\title{
Presión y área de contacto en reparación de manguito rotador: una revisión sistemática*
}

\section{Pressure and Contact Area in Rotator Cuff Repair: A Systematic Review}

\author{
Julio Contreras ${ }^{1,2,3}$ Rodrigo Liendo ${ }^{4,5}$ Cristóbal Maccioni ${ }^{3}$ Manuel Beltrán ${ }^{1,2}$
}

${ }^{1}$ Equipo de Hombro y Codo, Instituto Traumatológico, Santiago, Chile

${ }^{2}$ Departamento de Ortopedia y Traumatología, Universidad de Chile, Santiago, Chile

${ }^{3}$ Equipo de Hombro y Codo, Clínica BUPA, Santiago, Chile

${ }^{4}$ Equipo de Hombro y Codo, Pontificia Universidad Católica de Chile, Santiago, Chile

${ }^{5}$ Departamento de Ortopedia y Traumatología, Pontificia Universidad Católica de Chile, Santiago, Chile

\begin{abstract}
Dirección para correspondencia Julio José Contreras Fernández, MD, Equipo de Hombro y Codo, Instituto Traumatológico, San Martín 771, Santiago, RM, Chile (e-mail: Juliocontrerasmd@gmail.com).
\end{abstract}

Rev Chil Ortop Traumatol 2021;62(3):e208-e220.

\section{Resumen}

\section{Palabras Claves}

- manguito de los rotadores

- presión

- sutura

- técnicas de sutura

- traumatismos de los tendones

- tendones
Objetivo Proporcionar una sinopsis exhaustiva y un análisis de los estudios biomecánicos sobre la magnitud y distribución de la presión en la interfase tendónhuella de las roturas del manguito rotador, informadas en la literatura en los últimos cinco años.

Métodos La investigación se realizó de acuerdo con los métodos descritos en el Manual Cochrane. Los resultados se informan de acuerdo con el consenso de Ítems Preferidos de Reporte en Revisiones Sistemáticas y Metaanálisis (Preferred Reporting Items for Systematic Reviews and Meta-Analyses, PRISMA, en inglés). La búsqueda se realizó el $1^{\text {er }}$ de junio de 2020 . Se identificaron e incluyeron estudios ex vivo de ciencia básica y estudios biomecánicos publicados, que evaluaran la magnitud y distribución de la presión en la interfase tendón-huella de las roturas del manguito rotador reparadas entre enero de 2015 y junio de 2020. Se realizaron búsquedas sistemáticas en las bases de datos MEDLINE, Embase, Scopus y Google Scholar utilizando los términos y operadores booleanos: (Rotator Cuff OR Supraspinatus OR Infraspinatus OR Subscapularis OR Teres Minor) AND Pressure AND Footprint. En la base de datos Embase, respetando su sintaxis, se utilizó: Rotator Cuff AND Pressure AND Footprint.

Resultados Un total de 15 de los 87 artículos encontrados cumplieron con todos los criterios de elegibilidad y se incluyeron en el análisis.

* Trabajo realizado en el Instituto Traumatológico, Santiago, Chile.

recibido

28 de junio de 2020

aceptado

06 de agosto de 2021
DOI https://doi.org/

$10.1055 / \mathrm{s}-0041-1740142$ ISSN $0716-4548$. (c) 2021. Sociedad Chilena de Ortopedia y Traumatologia. All rights reserved.

This is an open access article published by Thieme under the terms of the Creative Commons Attribution-NonDerivative-NonCommercial-License, permitting copying and reproduction so long as the original work is given appropriate credit. Contents may not be used for commercial purposes, or adapted, remixed, transformed or built upon. (https://creativecommons.org/ licenses/by-nc-nd/4.0/)

Thieme Revinter Publicações Ltda., Rua do Matoso 170, Rio de Janeiro, RJ, CEP 20270-135, Brazil 


\begin{abstract}

\section{Keywords}

- rotator cuff

- pressure

- suture

- suture techniques

- tendon injuries

- tendons

Objective To provide a comprehensive synopsis and analysis of biomechanical studies on the magnitude and distribution of pressure at the tendon-footprint interface of rotator cuff tears reported in the literature in the last five years.

Methods The research was performed according to the methods described in the Cochrane Manual. The results are reported according to the Preferred Reporting Items for Systematic Reviews and Meta-Analyses (PRISMA) consensus. The search was performed on June $1^{\text {st }}, 2020$. We identified and included ex vivo basic science studies and published biomechanical studies that evaluated the magnitude and distribution of pressure at the tendon-footprint interface of rotator cuff tears repaired between January 2015 and June 2020. Systematic searches on the MEDLINE, Embase, Scopus and Google Scholar databases were performed using the terms and Boolean operators: (Rotator Cuff OR Supraspinatus OR Infraspinatus OR Subscapularis OR Teres Minor) AND Pressure AND Footprint. In the Embase database, respecting its syntax, the following was used: Rotator Cuff AND Pressure AND Footprint. Results In total, 15 of the 87 articles found fulfilled all the eligibility criteria and were included in the analysis.

Conclusion The pressure and contact area would be biomechanically optimized with an equivalent transosseous double-row repair, without knots in the medial row, and with the use of tapes for its execution, specific repair concepts for delaminated tears, and a limitation of abduction in the immediate postoperative period.
\end{abstract}

Conclusión La presión y área de contacto sería optimizada biomecánicamente con una reparación transósea de doble fila equivalente, sin nudos en la hilera medial, y con el uso de cintas para su ejecución, conceptos de reparación específica para roturas delaminadas, y limitación de la abducción en el postoperatorio inmediato.

\section{Introducción}

La reparación artroscópica del manguito rotador ha presentado un aumento constante en el último tiempo. ${ }^{1}$ Los resultados clínicos y funcionales son de buenos a excelentes en el corto y largo plazos en la mayoría de los casos; ${ }^{2-5}$ sin embargo, las tasas de rerotura siguen siendo considerables, descritas entre un $11 \%$ y un $68 \%$ en algunas series, incluso alcanzando un $94 \%$ en algunos estudios. ${ }^{6-8}$

La cirugía de reparación del manguito rotador busca establecer una interfase fibrovascular entre el tendón y la huella, que es necesaria para la curación y restauración de la inserción fibrocartilaginosa (entesis); para lograr esto, se busca un constructo que maximice el contacto presurizado entre el tendón y el hueso mientras mantiene la resistencia mecánica contra la carga fisiológica. ${ }^{9}$ La rerotura se asocia a factores del paciente y de la reparación (factores anatómicos). Respecto a los factores del paciente, se asocian a aumento de la edad, mayor tamaño de la rotura (compromiso de múltiples tendones), menor calidad tendínea, atrofia muscular, degeneración grasa (Goutallier $\geq 3$ ), retracción tendínea, mayor tiempo de evolución, y presencia de comorbilidades (tabaquismo, diabetes, hipercolesterolemia, alcoholismo, obesidad, e hipertensión). 7,10

Respecto a los factores anatómicos de la reparación, depende de la tensión del constructo, de la perfusión tisular, del micromovimiento en la interfase tendón-huella, y de la presión y área de contacto en la huella. ${ }^{11}$ El principio subyacente es que una mayor magnitud y distribución del área de contacto del tendón al hueso dará como resultado una mayor posibilidad de curación del tendón. ${ }^{12}$

Diversos estudios ${ }^{5,13}$ biomecánicos de la reparación en doble fila (DF) han demostrado un aumento de la resistencia a la falla por carga y una disminución de la formación de espacios (gap) en la interfase tendón-huella en comparación con la reparación de fila única (FU).

La técnica transósea equivalente (TOE; también llamada "puente de sutura") fue diseñada para mejorar la magnitud y distribución de la presión en la huella de las roturas del manguito rotador reparadas; los extremos de la sutura de la fila medial se colocan sobre el lado bursal del manguito rotador, y se aseguran al margen lateral de la huella con un ancla sin nudos. ${ }^{14,15}$

El objetivo de esta revisión sistemática es proporcionar una sinopsis exhaustiva y un análisis crítico de los estudios biomecánicos sobre la magnitud y distribución de la presión, en la interfase tendón-huella, de las roturas del manguito rotador, considerando diversos factores en la reparación, informadas en la literatura en los últimos cinco años.

\section{Materiales y Métodos}

La investigación se realizó de acuerdo con los métodos descritos en el Manual Cochrane. ${ }^{16}$ Los resultados se 
informan de acuerdo con el consenso de Ítems Preferidos de Reporte en Revisiones Sistemáticas y Metaanálisis (Preferred Reporting Items for Systematic Reviews and Meta-Analyses, PRISMA, en inglés). ${ }^{17}$ La búsqueda se realizó el $1^{\text {er }}$ de junio de 2020.

\section{Criterio de Elegibilidad}

Se identificaron e incluyeron estudios ex vivo de ciencia básica, y estudios biomecánicos publicados, que evaluaran la magnitud y distribución de la presión en la interfase tendón-huella de las roturas del manguito rotador reparadas entre enero de 2015 y junio de 2020, si cumplían con los siguientes criterios de inclusión: medición de presión y del área de contacto en la interfase tendón-huella, y descripción completa de la configuración de las pruebas biomecánicas, de las técnicas quirúrgicas, y de la metodología utilizada.

Fueron excluidos los estudios de resultados clínicos, tesis de investigación, resúmenes de congresos, artículos de técnicas quirúrgicas, y capítulos de libros.

\section{Búsqueda Bibliográfica}

Se realizó una revisión sistemática de la literatura para identificar todas las publicaciones de habla inglesa relacionadas con la evaluación biomecánica de la reparación del manguito rotador. Se realizaron búsquedas sistemáticas en las bases de datos MEDLINE, Embase, Scopus y Google Scholar, utilizando los términos y operadores booleanos: (Rotator Cuff OR Supraspinatus OR Infraspinatus OR Subscapularis OR Teres Minor) AND Pressure AND Footprint. En la base de datos Embase, respetando su sintaxis, se utilizó: Rotator Cuff AND Pressure AND Footprint. Cuatro revisores realizaron una selección independiente de títulos y resúmenes. Todos los artículos elegibles fueron referenciados manualmente para asegurar que se incluyeran otros estudios potenciales. Los desacuerdos fueron resueltos por consenso.

\section{Resultados}

\section{Selección de Estudios y Características}

En la búsqueda bibliográfica, identificamos 31 estudios para consideración; por el resumen, se excluyeron 2 por ser capítulos de libros. Finalmente, se excluyeron 14 estudios luego de la revisión de los manuscritos de texto completo, y sólo 15 de los artículos cumplieron con todos los criterios de elegibilidad y se incluyeron en el análisis (- Figura 1). ${ }^{18-32}$ En general, el acuerdo entre los revisores con respecto a la elegibilidad final fue excelente (no hubo desacuerdos). Los 15 estudios se publicaron en inglés entre 2015 y 2020. Las características importantes de los estudios se resumen en la - Tabla 1. Se agruparon los artículos revisados por temas relevantes: "Biomecánica de la fila medial en reparaciones de doble fila," "Comparación de cinta versus sutura" y "Características biomecánicas de distintas configuraciones".

\section{Biomecánica de la Fila Medial en Reparaciones de Doble Fila}

La preservación de la integridad estructural del manguito rotador, evitando nudos en tejidos dañados, mejoraría la cicatrización. ${ }^{33,34}$ Stone et al. ${ }^{31}$ midieron la fuerza de contacto y la presión en la interfase tendón-huella en un modelo de Sawbone (Vashon Island, WA, EEUU) graduado para estudios biomecánicos, utilizando un aloinjerto acelular de dermis humana para simular el tendón del manguito rotador; compararon un constructo DF con fila medial sin nudos versus fila medial con nudos, y no encontraron diferencias significativas. Cualitativamente, los autores ${ }^{31}$ describen que anudar la fila medial aumentó la presión de contacto focal y corrugó la periferia del constructo; sin embargo, este fenómeno no fue evaluado cuantitativamente, ni su impacto biológico. Estos resultados apoyan la hipótesis de que una fila medial sin nudo no reduce la fuerza de contacto total en un constructo de DF, favoreciendo los factores biológicos de la reparación desde un punto de vista teórico.

Sin embargo, en el contexto de una técnica de DF de puente de sutura, esta equivalencia biomecánica pudiese verse alterada, ya que los nudos en la fila medial pueden crear un efecto de tenodesis tal que, una vez que los nudos se hayan atado, el aumento de la carga (por tracción del manguito rotador) no conduciría a un "efecto de cuña" (wedge effect) del material de sutura, dejando de encajar el tendón sobre el hueso de la huella y perdiendo los efectos positivos de esto. ${ }^{35}$

Smith et al. ${ }^{30}$ evaluaron este "efecto de cuña" y el autorreforzamiento (self-reinforcement), comparando la presión de contacto en la interfase tendón-huella generada bajo cargas de tensión progresivas entre dos técnicas de reparación puente de sutura (con nudos y sin nudos en la fila medial). Los resultados de este estudio ${ }^{30}$ confirman que el autorreforzamiento ocurre en las reparaciones del manguito rotador DF de puente de sutura con y sin nudos de hilera medial; la carga de rendimiento se acercó a la carga de falla final, y la tasa de progresión de la compresión de la huella fue mayor en el grupo de reparación sin nudos, lo que proporcionó evidencia biomecánica de que el mecanismo de autorreforzamiento disminuye anudando la fila medial, probablemente asociado al efecto tenodesis; por lo que una reparación puente de sutura sin nudos mediales presenta iguales características de resistencia biomecánica, pero mejora la magnitud y la distribución de la presión en la interfase tendón-huella, lo que está asociado a menor plegamiento del tendón y a una teórica mejor irrigación del mismo, lo que favorecería en todos los aspectos la cicatrización del manguito rotador.

El año 2018, Smith y Lam, $^{29}$ usando un modelo biomecánico muy similar, pero enfocado a medir el efecto de la abducción del hombro a $-10^{\circ}, 0^{\circ}, 10^{\circ}, 20^{\circ}, 30^{\circ}, 40^{\circ}, 50^{\circ} \mathrm{y}$ $60^{\circ}$, encontraron que las presiones de contacto de la interfase tendón-huella y el autorreforzamiento son mayores en ángulos de abducción menores, tanto en técnicas anudadas como sin nudos. Esto tiene implicancias para la 


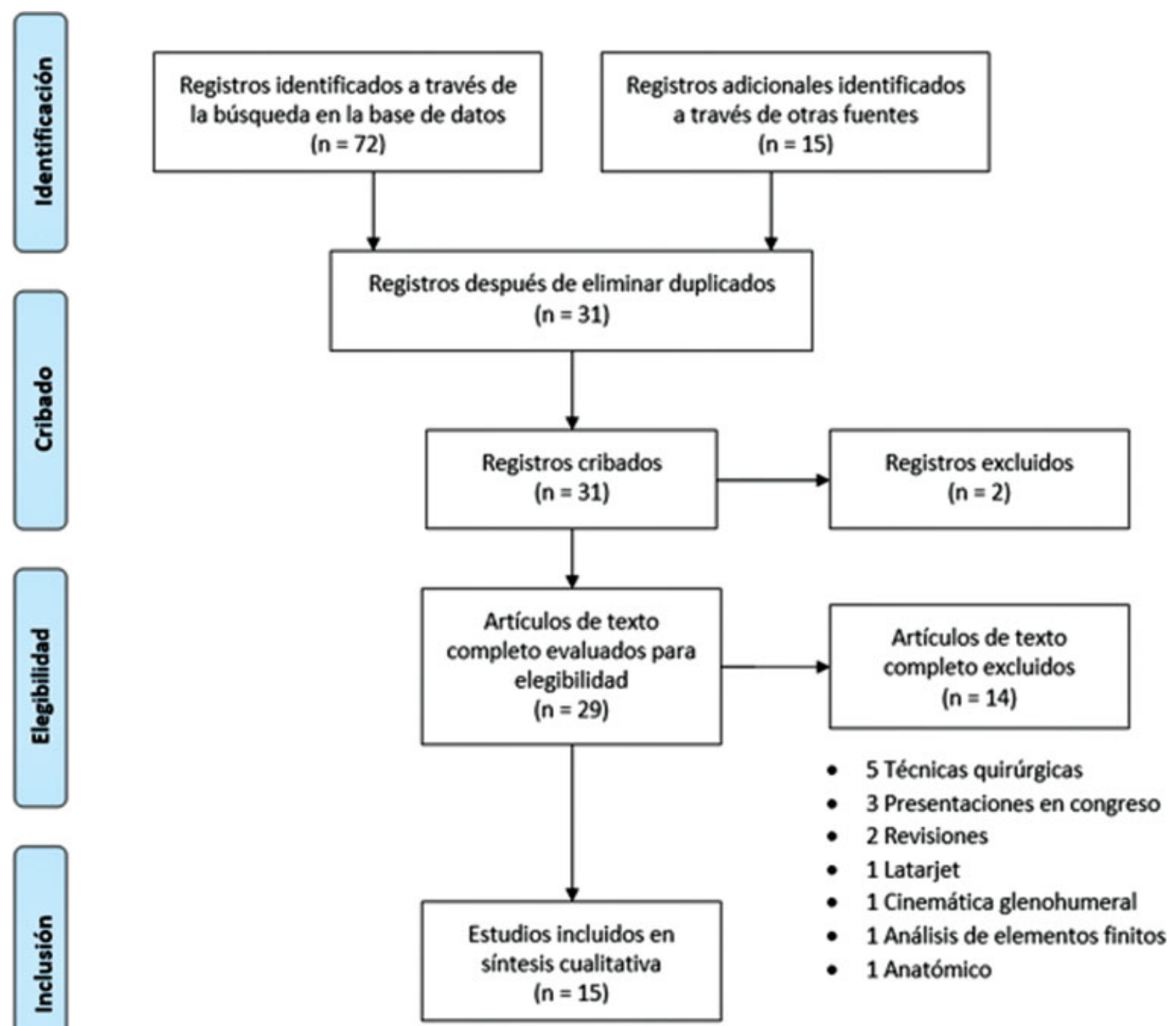

Fig. 1 Flujograma de los Ítems Preferidos a Reportar en Revisiones Sistemáticas y Metaanálisis ("Preferred Reporting Items for Systematic Reviews and Meta-Analyses," (PRISMA, en inglés). De los 87 registros iniciales, se incluyeron 15 estudios.

Tabla 1 Evaluaciones biomecánicas de los trabajos incluidos en esta revisión sistemática

\begin{tabular}{|c|c|c|c|}
\hline Autor Principal [ref] & $\mathrm{N}$ & Modelo & Evaluación Biomecánica \\
\hline \multirow[t]{3}{*}{ Caldow et al. ${ }^{18}$} & 56 & Cordero & Evaluación de distribución de presión con Fujifilm (Super-low) \\
\hline & & & Tensión de la reparación: $10 \mathrm{~N}$ \\
\hline & & & Además, se midió la carga de fallo \\
\hline \multirow[t]{3}{*}{ Dyrna et al. ${ }^{19}$} & 30 & Humano & Posición humeral: $0^{\circ}$ rotación y $0^{\circ}$ de abducción \\
\hline & & & Precarga: $10-100 \mathrm{~N}, 300$ ciclos a $0,5 \mathrm{~Hz}$ \\
\hline & & & $\begin{array}{l}\text { Se midió el área de contacto, la fuerza de contacto, la presión de contacto, el pico de la } \\
\text { presión de contacto, el modo de fallo }\end{array}$ \\
\hline \multirow[t]{4}{*}{ Huntington et al. ${ }^{20}$} & 60 & Cordero & Evaluación de distribución de presión con Fujifilm (Super-low) \\
\hline & & & Precarga 10N. Evaluación de la presión de la reparación se hizo con celda de carga Dynacell Instron \\
\hline & & & 200 ciclos, $10-62 \mathrm{~N}$ a $0,25 \mathrm{~Hz}$ \\
\hline & & & $\begin{array}{l}\text { Presión de contacto de huella, área de contacto, rigidez, resistencia a la tracción, modo de } \\
\text { fallo }\end{array}$ \\
\hline Kim et al. ${ }^{21}$ & 22 & Humano & Evaluación de distribución de presión con Fujifilm por 120 segundos postreparación \\
\hline
\end{tabular}


212 Biomecánica de reparación de manguito rotador Fernández et al.

Tabla 1 (Continued)

\begin{tabular}{|c|c|c|c|}
\hline Autor Principal [ref] & $\mathrm{N}$ & Modelo & Evaluación Biomecánica \\
\hline & & & Tensión de la reparación: $40 \mathrm{~N}$ \\
\hline \multirow[t]{5}{*}{ Liu et al. ${ }^{22}$} & 16 & Ovino & Posición humeral: $-10^{\circ}, 0^{\circ}$ y $10^{\circ}$ de abducción \\
\hline & & & Precarga de $30 \mathrm{~N}$ \\
\hline & & & La presión de contacto de la huella se midió a 10 N, 20 N y $30 \mathrm{~N}$ \\
\hline & & & Se midió con celda de carga Dynacell Instron \\
\hline & & & Además, se midió la carga de fallo \\
\hline \multirow[t]{4}{*}{ Liu et al. ${ }^{23}$} & 10 & Ovino & Posición humeral: $0^{\circ}, 20^{\circ}$ y $40^{\circ}$ de abducción \\
\hline & & & El autorreforzamiento se midió con cargas progresivas de $10 \mathrm{~N}$ a $60 \mathrm{~N}$ \\
\hline & & & Se midió con celda de carga Dynacell Instron \\
\hline & & & $\begin{array}{l}\text { Presión de contacto de huella, carga de fluencia, carga de tracción a falla, y energía máxima a } \\
\text { falla }\end{array}$ \\
\hline \multirow[t]{2}{*}{$\mathrm{Ng}$ et al. ${ }^{24}$} & 24 & Porcino & $\begin{array}{l}\text { Evaluación de distribución de presión con Fujifilm (Prescale Ultra Super Low Pressure) por } 60 \\
\text { segundos postreparación }\end{array}$ \\
\hline & & & Sin medición de la tensión de la reparación \\
\hline \multirow[t]{4}{*}{ Park et al. ${ }^{25}$} & 18 & Humano & $\begin{array}{l}\text { Posición humeral: } 30^{\circ} \text { de rotación externa, } 0^{\circ} \text { de rotación, } 30^{\circ} \text { de rotación interna; a } 0^{\circ} \text { y } 30^{\circ} \\
\text { de abducción }\end{array}$ \\
\hline & & & Fuerza de contacto, área y presión medidas con Tekscan 4041, carga de fallo \\
\hline & & & Tensiometría a $60 \mathrm{~N}, 90 \mathrm{~N}$ y $120 \mathrm{~N}$ de la técnica transósea equivalente \\
\hline & & & Carga tendínea a $30 \mathrm{~N}$ \\
\hline \multirow[t]{3}{*}{ Park et al. ${ }^{26}$} & 8 & Humano & Posición humeral: $0^{\circ}$ y $30^{\circ}$ de abducción \\
\hline & & & Fuerza de contacto, área y presión medidas con Tekscan 4041 \\
\hline & & & El autorreforzamiento se midió con cargas progresivas de $0 \mathrm{~N}$ a $60 \mathrm{~N}$ \\
\hline \multirow[t]{4}{*}{ Pauzenberger et al. ${ }^{27}$} & 18 & Humano & $\begin{array}{l}\text { Posición humeral: } 30^{\circ}-60^{\circ} \text { de rotación externa, } 0^{\circ} \text { de rotación, } 30^{\circ}-60^{\circ} \text { de rotación interna; a } \\
0^{\circ}, 30^{\circ} \text { y } 60^{\circ} \text { de abducción }\end{array}$ \\
\hline & & & $\begin{array}{l}\text { Precarga } 30 \text { N a } 50 \text { N. Evaluación de la presión de la reparación con sensor Tekscan Model } \\
4205\end{array}$ \\
\hline & & & 200 ciclos $10-100 \mathrm{~N}$ a $1 \mathrm{~Hz}$ \\
\hline & & & Se midió área de contacto, presión de contacto, modo de fallo \\
\hline \multirow[t]{4}{*}{ Simmer Filho et al. ${ }^{28}$} & 24 & Humano & Posición humeral: $0^{\circ}$ de rotación y $0^{\circ}$ y $30^{\circ}$ de abducción \\
\hline & & & Precarga: 30 N. Evaluación de la presión de la reparación con sensor Tekscan Model 4205 \\
\hline & & & $50 \mathrm{~N}$ por 30 segundos a $30 \mathrm{~N}$ por 30 segundos \\
\hline & & & Se midió área de contacto y presión de contacto \\
\hline \multirow[t]{4}{*}{ Smith et al. ${ }^{29}$} & 18 & Ovino & Posición humeral: $-10^{\circ}, 0^{\circ}, 10^{\circ}, 20^{\circ}, 30^{\circ}, 40^{\circ}, 50^{\circ}$ y $60^{\circ}$ de abducción \\
\hline & & & El autorreforzamiento se midió con cargas progresivas de $10 \mathrm{~N}$ a $60 \mathrm{~N}$ \\
\hline & & & Se midió con celda de carga Dynacell Instron \\
\hline & & & Presión de contacto de huella \\
\hline \multirow[t]{4}{*}{ Smith et al. ${ }^{30}$} & 18 & Ovino & Posición humeral: $0^{\circ}$ y $20^{\circ}$ de abducción \\
\hline & & & El autorreforzamiento se midió con cargas progresivas de $10 \mathrm{~N}$ a $60 \mathrm{~N}$ \\
\hline & & & Se midió con celda de carga Dynacell Instron \\
\hline & & & $\begin{array}{l}\text { Presión de contacto de huella, carga de fluencia, carga de tracción a falla, y energía máxima a } \\
\text { falla }\end{array}$ \\
\hline \multirow[t]{2}{*}{ Stone et al. ${ }^{31}$} & 60 & Sawbone & Posición humeral: $0^{\circ}$ de rotación y $0^{\circ}$ de abducción \\
\hline & & & Se midió área de contacto y presión de contacto con Tekscan (no especifica modelo) \\
\hline \multirow[t]{3}{*}{ Urch et al. ${ }^{32}$} & 10 & Humano & $\begin{array}{l}\text { Posición humeral: } 30^{\circ} \text { de rotación externa, } 0^{\circ} \text { de rotación, } 30^{\circ} \text { de rotación interna; a } 0^{\circ} \text { y } 30^{\circ} \\
\text { de abducción }\end{array}$ \\
\hline & & & Precarga: 25 N. Evaluación de la presión de la reparación con sensor Tekscan Model 4040 \\
\hline & & & $\begin{array}{l}\text { Se midió área de contacto, fuerza de contacto, presión de contacto y pico de presión de } \\
\text { contacto }\end{array}$ \\
\hline
\end{tabular}


Tabla 2 Configuración de la reparación de los trabajos incluidos en esta revisión sistemática

\begin{tabular}{|c|c|}
\hline Autores & Configuración de la reparación \\
\hline \multirow[t]{4}{*}{ Caldow et al. ${ }^{18}$} & $\begin{array}{l}\text { FU1: Sutura cruzada de una sola fila con puntos superpuestos orientados } 45^{\circ} \text { al tendón, } 2 \text { anclas } \\
\text { TwinFix, cargas con FiberWire \# } 2\end{array}$ \\
\hline & FU2: Sutura Mason-Allen de una sola fila, 2 anclas TwinFix, cargas con FiberWire \# 2 \\
\hline & $\begin{array}{l}\text { DF1: } 2 \text { puntos colchoneros mediales y } 2 \text { Mason-Allen laterales, } 4 \text { anclas TwinFix, cargas con FiberWire \# } \\
2\end{array}$ \\
\hline & TO: Reparación transósea con Fiberwire \# 2 \\
\hline \multirow[t]{7}{*}{ Dyrna et al. ${ }^{19}$} & Rotura $25 \%$ superior del subescapular \\
\hline & FU: $2 \times 4,5$ mm Bio-Corkscrew, doble carga \\
\hline & $\begin{array}{l}\text { DF híbrida: } 2 \times 4,75 \text { mm Bio-Swivelock ( } 1 \text { ancla superolateral y } 1 \text { ancla inferomedial; } 1 \text { lazo de } \\
\text { FiberTape) }\end{array}$ \\
\hline & Rotura $25 \%$ superior del subescapular \\
\hline & FU: $2 \times 4,5 \mathrm{~mm}$ Bio-Corkscrew, doble carga \\
\hline & $\begin{array}{l}\text { DF híbrida: } 2 \times 4,75 \text { mm Bio-Swivelock ( } 1 \text { ancla superolateral y } 1 \text { ancla inferomedial; } 1 \text { lazo de } \\
\text { FiberTape) }\end{array}$ \\
\hline & DF: $3 \times 4,75$ mm Bio-Swivelock ( 1 ancla superolateral y 2 anclas mediales; 2 lazos de FiberTape) \\
\hline \multirow[t]{4}{*}{ Huntington et al. ${ }^{20}$} & DF1: Puente de sutura con FiberWire \# 2 con 4 anclas sin nudo Bio-Swivelock \\
\hline & DF2: Puente de sutura con FiberTape con 4 anclas sin nudo Bio-Swivelock \\
\hline & DF3: Puente de sutura con FiberWire \# 2 con 3 anclas sin nudo Bio-Swivelock \\
\hline & DF4: Puente de sutura con FiberTape con 3 anclas sin nudo Bio-Swivelock \\
\hline \multirow[t]{2}{*}{ Kim et al. ${ }^{21}$} & TOE1: $2 \times 5,0$ mm Paladin medial, simple carga Hi-Fi \# 2 (hilera anudada) $+2 \times$ PopLok lateral, sin nudo \\
\hline & $\begin{array}{l}\text { TOE1: } 2 \times 5,0 \text { mm Paladin medial, simple carga Hi-Fi \# } 2 \text { (Mason-Allen modificado) }+2 \times \text { PopLok lateral, } \\
\text { sin nudo }\end{array}$ \\
\hline \multirow[t]{2}{*}{ Liu et al. ${ }^{22}$} & $\begin{array}{l}\text { BT1: Banda de tensión con } 2 \text { suturas FiberWire tipo colchonero invertido, a } 2 \text { anclas SwiveLock 5,5 mm } \\
\text { laterales, sin nudo }\end{array}$ \\
\hline & $\begin{array}{l}\text { BT2: Banda de tensión con } 2 \text { FiberTape tipo colchonero invertido, a } 2 \text { anclas SwiveLock 5,5 mm } \\
\text { laterales, sin nudo }\end{array}$ \\
\hline \multirow[t]{2}{*}{ Liu et al. ${ }^{23}$} & $\begin{array}{l}\text { DF1: } 2 \times 4,75 \mathrm{~mm} \text { SwiveLock medial, simple carga de FiberWire \# } 2 \text { (hilera anudada) }+2 \times 4,75 \mathrm{~mm} \\
\text { Bio-Swivelock lateral, sin nudo }\end{array}$ \\
\hline & $\begin{array}{l}\text { DF2: } 2 \times 4,75 \mathrm{~mm} \text { SwiveLock medial, simple carga de FiberTape (hilera sin nudo) }+2 \times 4,75 \mathrm{~mm} \text { Bio- } \\
\text { Swivelock lateral, sin nudo }\end{array}$ \\
\hline \multirow[t]{3}{*}{$\mathrm{Ng}$ et al. ${ }^{24}$} & $\begin{array}{l}\text { DF1: } 2 \times 5,5 \mathrm{~mm} \text { Bio-Corkscrew medial, doble carga de FiberWire \# } 2+2 \times 5,5 \mathrm{~mm} \text { Bio-Swivelock } \\
\text { lateral, sin nudo }\end{array}$ \\
\hline & $\begin{array}{l}\text { DF2: } 2 \times 5,5 \mathrm{~mm} \text { Bio-Corkscrew medial, doble carga de FiberWire \# } 2+1 \times 5,5 \mathrm{~mm} \text { Bio-Swivelock } \\
\text { lateral, sin nudo }\end{array}$ \\
\hline & $\begin{array}{l}\text { DF3: } 1 \times 5,5 \mathrm{~mm} \text { Bio-Corkscrew medial, doble carga de FiberWire \# } 2+2 \times 5,5 \mathrm{~mm} \text { Bio-Swivelock } \\
\text { lateral, sin nudo }\end{array}$ \\
\hline Park et al. ${ }^{25}$ & $\begin{array}{l}\text { TOE: } 2 \times 5,5 \mathrm{~mm} \text { Healix medial, simple carga de FiberWire \# } 2 \text { (hilera sin nudo) }+2 \times \text { Corkscrew lateral } \\
\text { (con pasador de sutura) }\end{array}$ \\
\hline \multirow[t]{2}{*}{ Park et al. ${ }^{26}$} & $\begin{array}{l}\text { TOE1: } 2 \times 5,5 \mathrm{~mm} \text { SwiveLock medial, simple carga de FiberTape (hilera anudada) }+2 \times 4,75 / 5,5 \mathrm{~mm} \\
\text { Bio-Swivelock lateral, sin nudo }\end{array}$ \\
\hline & $\begin{array}{l}\text { TOE2: } 2 \times 5,5 \mathrm{~mm} \text { SwiveLock medial, simple carga de FiberTape (hilera sin nudo) }+2 \times 4,75 / 5,5 \mathrm{~mm} \\
\text { Bio-Swivelock lateral, sin nudo }\end{array}$ \\
\hline \multirow[t]{3}{*}{ Pauzenberger et al. ${ }^{27}$} & $\begin{array}{l}\text { TOE de puente medial con nudos: } 2 \times 5,5 \mathrm{~mm} \text { Bio-Corkscrew medial, doble carga de FiberWire \# } 2 \\
\text { (mediales al cable, con nudo) }+2 \times 5,5 \mathrm{~mm} \text { Bio-Swivelock lateral, sin nudo }\end{array}$ \\
\hline & $\begin{array}{l}\text { TOE de puente medial sin nudos: } 2 \times 5,5 \mathrm{~mm} \text { Bio-Swivelock medial, carga simple de } \\
\text { FiberTape }+2 \times 5,5 \mathrm{~mm} \text { Bio-Swivelock lateral, sin nudo }\end{array}$ \\
\hline & $\begin{array}{l}\text { TOE de doble capa: } 2 \times 5,5 \mathrm{~mm} \text { Bio-Corkscrew medial, doble carga de FiberWire \# } 2 \text { (mediales al cable, } \\
1 \text { sutura pasada y } 1 \text { sutura loop articular) }+2 \times 5,5 \mathrm{~mm} \text { Bio-Swivelock lateral, sin nudo }\end{array}$ \\
\hline Simmer Filho et al. ${ }^{28}$ & FU1: $2 \times 4.5$ mm Bio-Corkscrew FT, doble carga de FiberWire \# 2 (nudos simples) \\
\hline
\end{tabular}


Tabla 2 (Continued)

\begin{tabular}{|c|c|}
\hline Autores & Configuración de la reparación \\
\hline & $\begin{array}{l}\text { FU2: Banda de tensión con } 2 \text { suturas FiberTape tipo colchonero invertido, a } 2 \text { anclas 4,75 mm Bio- } \\
\text { Composite SwiveLock }\end{array}$ \\
\hline & $\begin{array}{l}\text { FU3: Banda de tensión con } 2 \text { suturas FiberTape tipo colchonero invertido + FiberLink medial al } \\
\text { colchonero, a } 2 \text { anclas 4,75 mm Bio-Composite SwiveLock }\end{array}$ \\
\hline \multirow[t]{2}{*}{ Smith et al. ${ }^{29}$} & $\begin{array}{l}\text { DF1: } 2 \times 4,75 \mathrm{~mm} \text { SwiveLock medial, simple carga de FiberWire \# } 2 \text { (hilera anudada) }+2 \times 4,75 \mathrm{~mm} \\
\text { Bio-Swivelock lateral, sin nudo }\end{array}$ \\
\hline & $\begin{array}{l}\text { DF2: } 2 \times 4,75 \mathrm{~mm} \text { SwiveLock medial, simple carga de FiberWire \# } 2 \text { (hilera sin nudo) }+2 \times 4,75 \mathrm{~mm} \\
\text { Bio-Swivelock lateral, sin nudo }\end{array}$ \\
\hline \multirow[t]{2}{*}{ Smith et al. ${ }^{30}$} & $\begin{array}{l}\text { DF1: } 2 \times 4,75 \mathrm{~mm} \text { SwiveLock medial, simple carga de FiberWire \# } 2 \text { (hilera anudada) }+2 \times 4,75 \mathrm{~mm} \\
\text { Bio-Swivelock lateral, sin nudo }\end{array}$ \\
\hline & $\begin{array}{l}\text { DF2: } 2 \times 4,75 \mathrm{~mm} \text { SwiveLock medial, simple carga de FiberWire \# } 2 \text { (hilera sin nudo) }+2 \times 4,75 \mathrm{~mm} \\
\text { Bio-Swivelock lateral, sin nudo }\end{array}$ \\
\hline \multirow[t]{4}{*}{ Stone et al. ${ }^{31}$} & $\begin{array}{l}\text { DF1: } 2 \times 5,5 \text { mm PEEK Healicoil medial, doble carga de FiberWire \# } 2 \text { (con nudos) + fila lateral } \\
\text { transósea }+ \text { aloinjerto acelular de dermis humana matrix HD }\end{array}$ \\
\hline & $\begin{array}{l}\text { DF2: } 2 \times 5,5 \text { mm PEEK Healicoil medial, doble carga de FiberWire \# } 2 \text { (sin nudos) + fila lateral } \\
\text { transósea + aloinjerto acelular de dermis humana matrix HD }\end{array}$ \\
\hline & $\begin{array}{l}\text { DF3: } 2 \times 5,5 \mathrm{~mm} \text { PEEK Healicoil medial, doble carga de FiberTape y sutura (con nudos) + fila lateral } \\
\text { transósea + aloinjerto acelular de dermis humana matrix HD }\end{array}$ \\
\hline & $\begin{array}{l}\text { DF4: } 2 \times 5,5 \text { mm PEEK Healicoil medial, doble carga de FiberTape y sutura (sin nudos) + fila lateral } \\
\text { transósea }+ \text { aloinjerto acelular de dermis humana matrix HD }\end{array}$ \\
\hline \multirow[t]{2}{*}{ Urch et al. ${ }^{32}$} & TOE Clásica: $2 \times 5,5 \mathrm{~mm}$ ancla medial, doble carga de FiberWire \# $2+2 \times 4,75 \mathrm{~mm}$ lateral, sin nudo \\
\hline & $\begin{array}{l}\text { TOE aumentada con fijación de borde lateral: } 2 \times 5,5 \mathrm{~mm} \text { ancla medial, doble carga de FiberWire \# } \\
2+2 \times 4,75 \mathrm{~mm} \text { lateral, sin nudo }+2 \text { suturas en bucle del borde lateral }\end{array}$ \\
\hline
\end{tabular}

Abreviaturas: BT, banda de tensión; DF, doble fila; FU, fila única; TO, transóseo; TOE, técnica transósea equivalente; PEEK, polieteretercetona.

rehabilitación después de la reparación del manguito rotador en DF.

Park et al. ${ }^{26}$ midieron el efecto de los nudos de la fila medial en el autorreforzamiento y el contacto de la huella. Las variables de prueba incluyeron $0^{\circ}$ y $30^{\circ}$ de abducción humeral. Este estudio demostró que los nudos de la fila medial inhibían el autorreforzamiento; en particular, los nudos mediales no mejoraron el contacto de la huella. Tales nudos causan efectivamente la tenodesis de la reparación, lo que impide el autorreforzamiento del tendón lateral y concentra el estrés en la fila medial; la carga del tendón no se transmite fácilmente, y no se convierte en una fuerza de compresión sobre la huella reparada. Esto podría proporcionar la justificación biomecánica que explica un mecanismo para las fallas mediales.

La reparación TOE muestra una mayor tasa de cicatrización en la interfase tendón-huella en comparación con la reparación $\mathrm{FU}^{5}{ }^{5,13}$ sin embargo, después de la reparación TOE, existe un patrón de rerotura único; en muchos casos, las fallas ocurrieron en la fila medial con un tendón bien adherido en la tuberosidad mayor, a pesar de que se obtuvo una cicatrización satisfactoria en el sitio de reparación. ${ }^{26}$ La posible sobretensión y estrangulamiento en la fila medial anudada puede dejar el tendón reparado vulnerable a una rerotura. ${ }^{26}$
La tensión es un factor importante para considerar con tal de no estrangular y disminuir la perfusión tisular del tendón, lo que trae consecuencias negativas para la cicatrización. ${ }^{36,37}$ En un modelo de reparación TOE con tensión variable y medida, Park et al. ${ }^{25}$ lograron demostrar como un incremento de la tensión de la puente de sutura de $60 \mathrm{~N}$ a $120 \mathrm{~N}$ generó un aumento significativo de la fuerza de contacto, de la presión máxima, y de la presión media en la interfase tendón-huella en todas las posiciones. Sin embargo, con respecto al área de contacto, aunque hubo diferencias significativas entre $60 \mathrm{~N}$ y $90 \mathrm{~N}$, excepto para 1 posición (abducción de $30^{\circ}$ y rotación externa de $30^{\circ}$ ), no se observaron diferencias significativas entre $90 \mathrm{~N}$ y $120 \mathrm{~N}$. Por lo tanto, los datos sugieren que la tensión de la puente de sutura sobre $90 \mathrm{~N}$ no tiene ningún beneficio aparente en este modelo cadavérico a tiempo cero, lo que se correlaciona con la innecesaria sobretensión en algunos constructos.

Kim et al. ${ }^{21}$ evaluaron si la reparación TOE sin nudo medial, utilizando una configuración tipo Mason-Allen modificada, proporcionaría un área de contacto y presión de interfase tendón-huella comparable con la reparación TOE con nudo medial convencional. La reparación TOE convencional mostró un área de contacto y presión de interfase significativamente mayores que la reparación de TOE sin nudos medial, medidas con película sensible a la presión. Estos resultados probablemente se asocian al tipo de 
medición, pero habría que tener en cuenta que la técnica Mason-Allen no agregaría más beneficio que sólo dejar sin nudo.

En este sentido, evaluando el autorreforzamiento y el evitar anudar, pero en reparaciones de FU, Simmer Filho et al. $^{28}$ compararon dos variaciones de reparaciones sin nudos de FU (reparación sin nudos y reparación rip-stop sin nudos) con una reparación con nudos de FU, enfocada en evaluar la presión de contacto y el área de contacto. Los hallazgos más importantes fueron que, bajo carga de tensión, ambas técnicas sin nudos de FU mostraron una mejor cobertura de huella y un área de contacto más grande en comparación con la técnica de nudos de FU. Además, ambas técnicas sin nudos también mostraron patrones de distribución de presión más uniformes.

\section{Comparación de Cinta versus Sutura}

La cinta es típicamente un material de sutura trenzado plano utilizado principalmente en reparaciones sin nudos, y, debido a su ancho prominente, tiene el potencial de reducir la incidencia de extracción del tendón de sutura en relación con la sutura tradicional, al tiempo que aumenta la resistencia del constructo en la huella. ${ }^{38}$ De Carli et al. ${ }^{39}$ fueron de los primeros en plantear un estudio biomecánico que demostrará una mayor estabilidad del constructo.

El primer estudio en examinar los resultados biomecánicos y clínicos de la reparación del manguito rotador con cinta más gruesa fue conducido Liu et al. ${ }^{22}$ el año 2017. En la parte biomecánica de este estudio, compararon el efecto sobre la presión de contacto en la interfase tendón-huella de dos constructos de banda de tensión, sólo cambiando FiberWire (Arthrex, Naples, FL, EEUU) por FiberTape (Arthrex). La reparación del manguito rotador con cinta mostró una presión de contacto de huella 3 veces mayor en comparación con la reparación con sutura $\left(0^{\circ}\right.$ de rotación con una carga de $30 \mathrm{~N}$ ). La carga de falla final del grupo de reparación de cinta fue significativamente mayor (1,5 veces) que la del grupo de sutura.

Huntington et al. ${ }^{20}$ compararon la presión de contacto, el área y la resistencia mecánica entre sutura y cinta. Encontraron mayor resistencia a la tracción en constructos de reparación con cinta. Sin embargo, encontraron mayor resistencia a la tracción máxima usando cinta sólo en aquellos constructos con cuatro anclas (en comparación con los de tres anclas). La rigidez no mostró diferencias significativas entre la cinta y la sutura en ambos grupos de reparación.

Liu et al. ${ }^{23}$ evaluaron biomecánicamente el autorreforzamiento comparando reparaciones con distintos materiales de suturas. En ambos materiales, observaron aumentos progresivos en la presión de contacto cuando la tensión aplicada aumentaba. El autorreforzamiento fue mayor con FiberTape sin nudos (mayor tasa de aumento en la compresión de la huella). A pesar de una mayor carga de rendimiento en el grupo del FiberTape, la diferencia entre la carga de rendimiento y la carga final fue similar en las dos técnicas. Además, los resultados de este estudio ${ }^{23}$ confirman que la abducción del hombro disminuye el autorreforzamiento en ambos constructos, aunque este efecto fue menos marcado en el grupo del FiberTape.

\section{Características Biomecánicas de Distintas Configuraciones}

Si bien muchos estudios han analizado las propiedades biomecánicas y los resultados de la reparación de FU o DF, pocos ${ }^{24}$ han comparado los diferentes tipos de configuraciones de DF, particularmente en el área de contacto en la interfase tendón-huella.

$\mathrm{Ng}$ y Tan, ${ }^{24}$ utilizando tendón de infraespinoso en un modelo porcino, compararon la distribución de presión en tres configuraciones de DF (TOE; dos anclas mediales y una lateral; y un ancla medial y dos laterales), y demostraron que esta técnica no sólo da como resultado una buena área de contacto en la huella (mayor de $70 \%$ de compresión), sino también que el uso de una configuración de 3 anclas en comparación con 4 anclas produce un área de contacto similar en la huella en roturas medianas (no mayores de $1,5 \mathrm{~cm} \times 2,5 \mathrm{~cm})$.

Un tema relevante para considerar es la formación de espacios ( $g a p$ ) en el borde anterior de la reparación TOE con la rotación humeral. ${ }^{40}$ Se ha demostrado que las rotaciones interna y externa afectan de manera diferencial la tensión en las regiones anterior y posterior de la reparación. ${ }^{32}$

Urch et al. ${ }^{32}$ evaluaron las presiones de contacto al agregar dos bucles de sutura al borde lateral libre del tendón e incluirlo en el ancla sin nudo lateral (configuración en "etiqueta de equipaje"), y demostraron presiones mayores (diferencia promedio $=23,1 \mathrm{kPa}$ ) en comparación con la construcción clásica TOE. Además, la configuración "etiqueta de equipaje" presentó presiones de contacto significativamente mayores a $30^{\circ}$ de rotación interna y $30^{\circ}$ de rotación externa en $0^{\circ}$ y $30^{\circ}$ de abducción. Finalmente, respecto al área de contacto, no hubo diferencias estadísticamente significativas en ninguna de las condiciones de prueba.

Respecto al tendón subescapular, su porción superior ha tomado relevancia biomecánica últimamente. ${ }^{19,41}$ Yoo et al. $^{42}$ investigaron esto en detalle, destacaron la importancia del borde lateral superior del tendón subescapular, debido a que comúnmente representa el sitio de rotura inicial con progresión inferior, y introdujeron el término "borde de ataque" (leading edge). Dyrna et al. ${ }^{19}$ analizaron tres configuraciones de reparación (FU con dos anclas; DF híbrida con un ancla superolateral; y DF con dos anclas mediales y un ancla superolateral) en un modelo de rotura de subescapular de $25 \%$ y $50 \%$ superior. La cobertura de la huella con respecto al tamaño del defecto no presentó diferencias significativas. Respecto a la cobertura y reconstrucción del "borde de ataque" del subescapular, se observaron diferencias significativas entre la construcción de FU y una construcción que utiliza un ancla colocada superolateralmente a favor de esta última, independientemente del tamaño de la rotura y el número de anclas utilizado.

La delaminación se describe como un desgarro horizontal entre las capas del manguito de los rotadores que resulta en 
isquemia local, revestimiento similar a la sinovial, aumento del movimiento entre capas, progresión de desgarro, y biomecánica alterada. ${ }^{43}$ La prevalencia de la delaminación en roturas extensas del manguito rotador oscila entre $38 \%$ y $88 \%$ en la literatura, ${ }^{44}$ mientras que la delaminación se ha identificado como un factor pronóstico negativo en las reparaciones del manguito rotador. ${ }^{45}$

Sin embargo, las técnicas comunes de reparación de DF ignoran la estructura multicapa del manguito rotador, y no logran restaurar la inserción de la cápsula articular superior y el tendón. Dicha reconstrucción no anatómica puede dar como resultado un desajuste de la tensión del tendón, alteraciones desfavorables de la biomecánica de la articulación glenohumeral y, en última instancia, falla de la reparación. $^{27}$ Pauzenberger et al. ${ }^{27}$ compararon las configuraciones de reparación de manguito rotador de DF masivas que se utilizan ampliamente (TOE con FiberWire y TOE con FiberTape), y una técnica de reparación específica de doble capa, la cual proporcionó un área de contacto mayor y una restauración superior de la huella a $60^{\circ}$ de la abducción glenohumeral; además, demostró un desplazamiento bajo carga cíclica comparable con el tendón nativo. La carga máxima en la falla fue comparable entre las construcciones de reparación. Por lo tanto, es probable que estas reparaciones tradicionales masivas no proporcionan realmente la restauración anatómica del manguito rotador y la inserción de la cápsula o las condiciones de tensión nativas. Estos resultados sugieren que la reparación específica de doble capa sin nudos podría restaurar anatómicamente la restricción estática proporcionada por la cápsula articular superior y el aspecto dinámico del manguito rotador facilitado por la capa del tendón del lado bursal, al tiempo que combina los beneficios de configuraciones de reparación sin nudos con la fuerza de fijación de una reparación TOE anudada medialmente. ${ }^{27}$

La reparación de Mason-Allen exhibe una mayor resistencia a la tracción en comparación con las reparaciones simples y de colchoneros, mientras que la resistencia final de una reparación de DF ha demostrado ser significativamente mayor. ${ }^{46,47}$

Caldow et al. ${ }^{18}$ evaluaron el área de contacto, la presión de contacto, la resistencia a la tracción, y la rigidez de una nueva reparación de sutura cruzada de una FU, y compararon los resultados con los de tres reparaciones ampliamente reportadas y establecidas (Mason-Allen, TOE y DF). La reparación de la sutura cruzada consiste en dos puntos superpuestos orientados $45^{\circ}$ al tendón, que actúan para aumentar el contacto tendón-huella y evitar el deslizamiento del tendón.

El resultado más significativo de este estudio ${ }^{18}$ fue que esta nueva técnica de reparación de sutura cruzada de una FU mejora el área de contacto presurizada en relación con la reparación de Mason-Allen, y da como resultado una presión de contacto media comparable en relación con las reparaciones de Mason-Allen y de DF. El estudio de Caldow et al. ${ }^{18}$ mostró que la reparación de la sutura cruzada produjo un área de contacto de huella $66 \%$ mayor en comparación con la reparación de Mason-Allen, y una resistencia a la tracción final similar a la de la reparación de Mason-Allen. La reparación de DF tenía una resistencia a la tracción máxima significativamente mayor que la de la sutura cruzada, la de Mason-Allen, y la de las reparaciones TOEs.

\section{Discusión}

La cirugía de reparación del manguito rotador busca establecer una interfase fibrovascular entre el tendón y la huella, que es necesaria para la cicatrización. ${ }^{9}$ La rerotura se asocia a factores del paciente y de la reparación, siendo la presión y el área de contacto en la interfase tendón-huella factores dependientes de la técnica quirúrgica escogida y de su ejecución por el cirujano.

Diversos estudios ${ }^{5,13}$ biomecánicos de la reparación en DF han demostrado un aumento de la resistencia a la falla por carga, áreas de contacto y presiones mejoradas, y una disminución de la formación de espacios (gaps) en la interfase tendón-huella en comparación con la reparación de FU. Sin embargo, las diversas configuraciones de reparación DF, como ha sido analizado en esta revisión, tienen un impacto directo en la presión y en el área de contacto en la interfase tendón-huella.

El autorreforzamiento es un mecanismo descrito por Burkhart et al. ${ }^{35}$ el año 2009, por el cual el aumento de la tensión aplicada al constructo genera una resistencia creciente a la falla estructural al generar un aumento progresivo de las fuerzas de compresión en la huella del tendón. Las fuerzas de compresión creadas en la huella aumentan la resistencia a la fricción entre el tendón y el hueso, reduciendo así la formación de espacios entre las dos superficies. $^{29,30,35}$

Se han propuesto tres mecanismos biomecánicos para explicar el autorreforzamiento en la reparación del manguito rotador. $^{35}$ Dos de estos se basan en un estrechamiento o acuñamiento del ángulo entre el material de sutura y el hueso a medida que el tendón se tensiona progresivamente. En la reparación TOE, la forma del constructo de la puente de sutura (mirada en un plano coronal; - Fig. 2a) cambia de rectangular a trapezoidal a medida que aumenta la carga de tracción. ${ }^{35}$ Esto provoca una deformación elástica del tendón, creando una fuerza de compresión perpendicular a la superficie del hueso, que aumenta a medida que aumenta la carga de tracción (-Fig. 2b). ${ }^{29,30,35}$

Un segundo mecanismo en las reparaciones DF TOE es el estrechamiento o acuñamiento del ángulo entre el material de sutura superior y el hueso a medida que aumentan las cargas de tracción. El material de sutura luego engancha el tendón más firmemente contra el hueso, aumentando la compresión de la huella. Esto se ha denominado el "efecto cuña" (- Fig. 3). ${ }^{35}$

Puede existir un mecanismo similar en la reparación de una FU en la que el asa de sutura se alarga bajo carga. La extremidad superior crea un vector de compresión relativa y un efecto denominado cuña de bucle focal (focal loop wedge effect $)^{48}$ (- Fig. 4). 

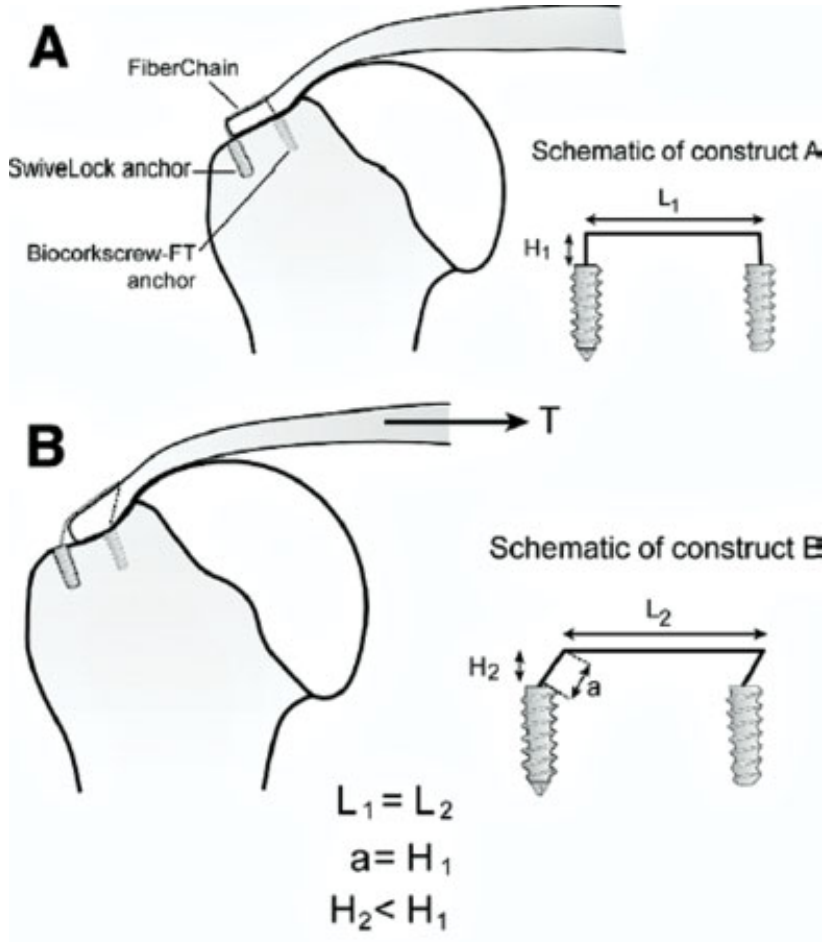

Fig. 2 (A) Esquema de la configuración de reparación SwiveLock y FiberChain (Arthrex) de doble fila previo a aplicación de carga (abrevituras: H1, grosor del manguito rotador antes de cargar; L1, longitud del tendón debajo de FiberChain). (B) Esquema de la configuración de reparación después de la carga (abreviaturas: T, fuerza de carga de tracción; L2, longitud del tendón debajo de FiberChain; a, longitud de FiberChain entre el borde del tendón y el ancla lateral; $\mathrm{H} 2$, espesor del manguito rotador comprimido bajo carga de tracción). Reproducido con permiso de Burkhart et al. ${ }^{35}$

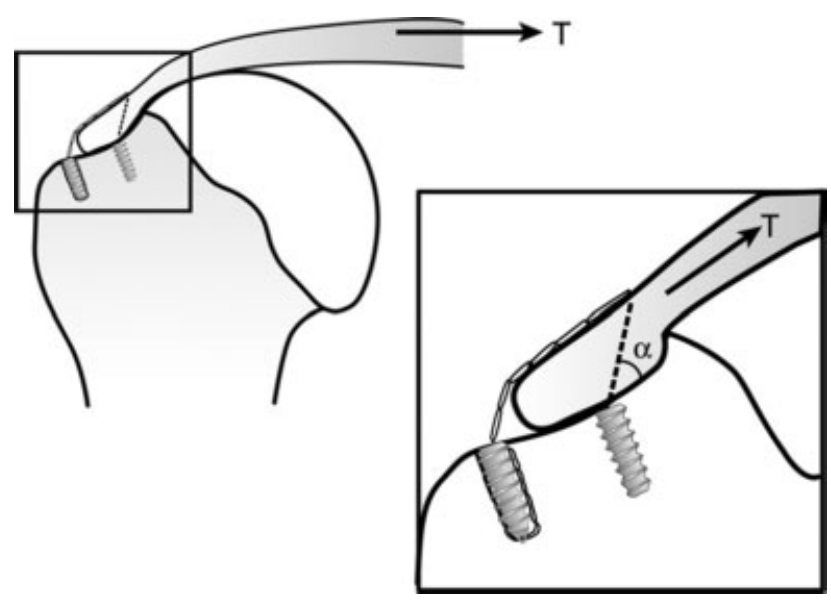

Fig. 3 Después de cargar el tendón: efecto de cuña de FiberChain (Arthrex) en el tendón. A medida que aumenta la carga (T), el ángulo $(\alpha)$ disminuye, acuñando el tendón más firmemente entre FiberChain y el hueso. Reproducido con permiso de Burkhart et al. ${ }^{35}$

Finalmente, un mayor ancho de sutura también puede aumentar el autorreforzamiento, ya que el material de diámetro más ancho tiene una mayor área de superficie en contacto con el tendón, lo que aumenta los otros dos mecanismos. $^{35}$

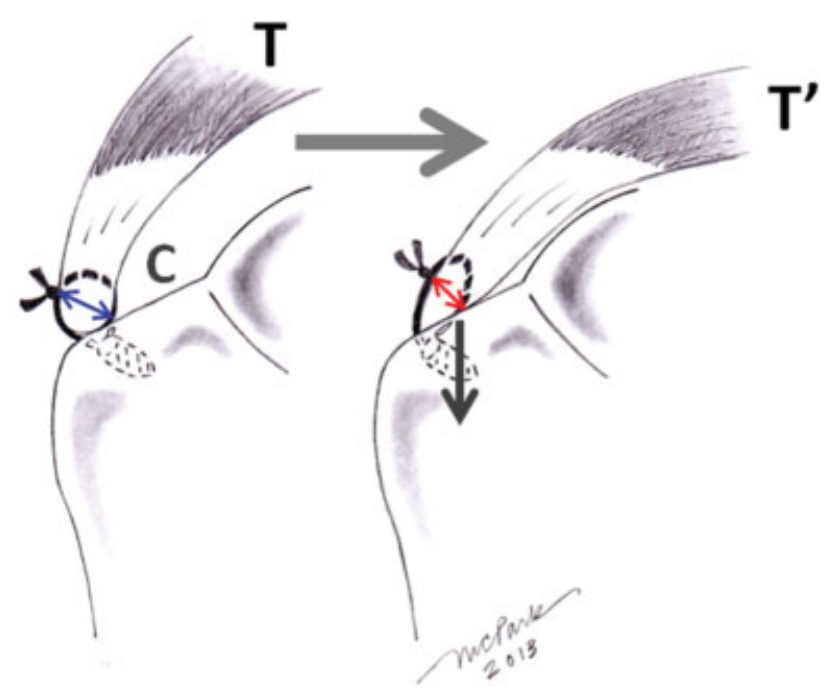

Fig. 4 La representación esquemática muestra como el contacto de la huella aumenta al aumentar la carga del tendón. A medida que la carga del tendón aumenta de T a T', el bucle de sutura de una reparación que no se fija medialmente se alarga y se estrecha (flechas dobles), creando una "cuña de bucle focal". Este efecto crea un vector de compresión sobre la huella lateralmente, y el área de contacto expuesta (C) disminuye. Con la fijación medial y las suturas de puente tendinoso, el efecto de "cuña" puede incluir toda la huella medial. Reproducido con permiso de Park et al. ${ }^{48}$

Fig. 5 Con base en los estudios biomecánicos revisados, podríamos concluir que los constructos que mejoran la presión y área de contacto en la interfase tendón-huella incluyen: a) reparación de doble fila transósea equivalente; b) sin nudos en la hilera medial; c) uso de cintas; d) conceptos de reparación específica para roturas delaminadas; y e) limitación de la abducción en el postoperatorio inmediato.

Smith et al. ${ }^{30}$ confirmaron que el autorreforzamiento ocurre en las reparaciones del manguito rotador DF TOE con y sin nudos de hilera medial; la carga de rendimiento se acercó a la carga de falla final, y la tasa de progresión de la compresión de la huella fue mayor en el grupo de reparación sin nudos, lo que proporcionó evidencia biomecánica de que el mecanismo de autorreforzamiento disminuye anudando la 
fila medial, probablemente asociado al efecto de tenodesis; por lo que una reparación TOE sin nudos mediales presenta iguales características de resistencia biomecánica, pero mejora la magnitud y la distribución de la presión en la interfase tendón-huella, asociadas a menor plegamiento del tendón y a una teórica mejor irrigación del mismo, lo que favorecería en todos los aspectos la cicatrización del manguito rotador.

Considerando los trabajos analizados, el uso de una configuración TOE, con cinta y sin nudos en la hilera medial, probablemente se asocia a las mejores condiciones biomecánicas de presión y área de contacto en la interfase tendón-huella. Además, durante el proceso de deslizamiento de nudos en la reparación artroscópica y la sobretensión de estos, puede producir pequeñas roturas en el tejido blando y generar una alta concentración de estrés. Además, se ha informado que la sutura trenzada tiene mayores propiedades abrasivas a través del tejido del manguito rotador en comparación con las suturas de monofilamento. ${ }^{22}$ La cinta ha demostrado una mayor carga media al fallo y una mayor área de contacto en la interfaz del tendón de la sutura, lo que facilita una distribución uniforme de la presión. ${ }^{22}$ Mook et al. ${ }^{49}$ presentaron en detalle esta técnica quirúrgica con concepto autorreforzante, con excelentes resultados según la opinión de los autores. Sin embargo, un factor relevante a considerar es la irrigación del tendón, y como esta puede comprometerse con una compresión aumentada en la interfase tendón-huella. Kim et al. ${ }^{50}$ analizaron la fuerza biomecánica y los resultados histológicos en un modelo de conejo, y demostraron que la falla de la fila medial (rotura intrasustancia) fue más frecuente en un constructo TOE con nudos, atribuible a un compromiso microvascular (menor número de vasos en la histología). Lamentablemente, no hay trabajos que comparen el efecto sobre la irrigación de usar una configuración sin nudos en la fila medial, y los beneficios son solamente teóricos.

Un punto interesante para considerar es la delaminación del manguito rotador. Se describe como un desgarro horizontal entre las capas ${ }^{43}$ con una prevalencia que ha oscilado entre $38 \%$ y $88 \% .{ }^{44}$ Pauzenberger et al. ${ }^{27}$ demostraron que una reparación específica de doble capa sin nudos podría restaurar anatómicamente la restricción estática proporcionada por la cápsula articular superior, y el aspecto dinámico del manguito rotador facilitado por la capa del tendón del lado bursal, al tiempo que combina los beneficios de configuraciones de reparación sin nudos con la fuerza de fijación de una reparación TOE anudada medialmente. Por lo tanto, probablemente, la sola aplicación de una reparación TOE no es suficiente para optimizar los resultados, y debe agregarse un gesto a la lámina articular, buscando la perfección en la técnica ejecutada.

Finalmente, hay que considerar el efecto de la abducción en los distintos tipos de constructos. En general, los trabajos analizados muestran que la abducción no afectó el autorreforzamiento para filas mediales anudadas, de acuerdo con la idea de que estas pueden causar tenodesis de la reparación, inhibiendo el mecanismo de autorreforzamiento. Para la reparación sin nudos, la abducción disminuye significativamente el autorreforzamiento; esto debiese ser considerado para la indicación de movilidad pasiva y el tipo de inmovilización durante las primeras semanas postoperatorias.

\section{Conclusión}

La presión y el área de contacto en la interfase tendón-huella es un factor dependiente de la técnica quirúrgica escogida y de su ejecución por el cirujano. Con base en los estudios biomecánicos revisados, podríamos concluir que los constructos que mejoran estos aspectos son aquellos que incluyen una reparación de DF TOE, sin nudos en la hilera medial, y que favorecen el uso de cintas para su ejecución, conceptos de reparación específica para roturas delaminadas, y limitación de la abducción en el postoperatorio inmediato.

\section{Conflicto de Intereses}

Los autores no tienen conflicto de intereses que declarar.

\section{Agradecimientos}

A nuestra familia, por el constante apoyo en nuestra labor de investigación.

\section{Referencias}

1 Jancuska J, Matthews J, Miller T, Kluczynski MA, Bisson LJ. A Systematic Summary of Systematic Reviews on the Topic of the Rotator Cuff. Orthop J Sports Med 2018;6(09): 2325967118797891. Doi: 10.1177/2325967118797891

2 Collin P, Colmar M, Thomazeau H, et al. Clinical and MRI Outcomes 10 Years After Repair of Massive Posterosuperior Rotator Cuff Tears. J Bone Joint Surg Am 2018;100(21):1854-1863. Doi: 10.2106/JBJS.17.01190

3 Collin P, Thomazeau H, Walch G, et al. Clinical and structural outcome twenty years after repair of isolated supraspinatus tendon tears. J Shoulder Elbow Surg 2019;28(01):196-202. Doi: 10.1016/j.jse.2018.07.023

4 Piper CC, Hughes AJ, Ma Y, Wang H, Neviaser AS. Operative versus nonoperative treatment for the management of full-thickness rotator cuff tears: a systematic review and meta-analysis. J Shoulder Elbow Surg 2018;27(03):572-576. Doi: 10.1016/j. jse.2017.09.032

5 Rossi LA, Rodeo SA, Chahla J, Ranalletta M. Current Concepts in Rotator Cuff Repair Techniques: Biomechanical, Functional, and Structural Outcomes. Orthop J Sports Med 2019;7(09): 2325967119868674. Doi: 10.1177/2325967119868674

6 Chona DV, Lakomkin N, Lott A, et al. The timing of retears after arthroscopic rotator cuff repair. J Shoulder Elbow Surg 2017;26 (11):2054-2059. Doi: 10.1016/j.jse.2017.07.015

7 Haque A, Pal Singh H. Does structural integrity following rotator cuff repair affect functional outcomes and pain scores? A metaanalysis. Shoulder Elbow 2018;10(03):163-169. Doi: $10.1177 / 1758573217731548$

8 Galatz LM, Ball CM, Teefey SA, Middleton WD, Yamaguchi K. The outcome and repair integrity of completely arthroscopically repaired large and massive rotator cuff tears. J Bone Joint Surg Am 2004;86(02):219-224. Doi: 10.2106/00004623-20040200000002

9 Jensen PT, Lambertsen KL, Frich LH. Assembly, maturation, and degradation of the supraspinatus enthesis. J Shoulder Elbow Surg 2018;27(04):739-750. Doi: 10.1016/j.jse.2017.10.030 
10 Desmoineaux P. Failed rotator cuff repair. Orthop Traumatol Surg Res 2019;105(1S):S63-S73. Doi: 10.1016/j.otsr.2018.06.012

11 Cicak N, Klobucar H, Bicanic G, Trsek D. Arthroscopic transosseous suture anchor technique for rotator cuff repairs. Arthroscopy 2006;22(05):565.e1-565.e6. Doi: 10.1016/j.arthro.2005.07.029

12 Park MC, Cadet ER, Levine WN, Bigliani LU, Ahmad CS. Tendonto-bone pressure distributions at a repaired rotator cuff footprint using transosseous suture and suture anchor fixation techniques. Am J Sports Med 2005;33(08):1154-1159. Doi: $10.1177 / 0363546504273053$

13 Hohmann E, König A, Kat CJ, Glatt V, Tetsworth K, Keough N. Single- versus double-row repair for full-thickness rotator cuff tears using suture anchors. A systematic review and metaanalysis of basic biomechanical studies. Eur J Orthop Surg Traumatol 2018;28(05):859-868. Doi: 10.1007/s00590-0172114-6

14 Park MC, Elattrache NS, Ahmad CS, Tibone JE. "Transosseousequivalent" rotator cuff repair technique. Arthroscopy 2006;22 (12):1360.e1-1360.e5. Doi: 10.1016/j.arthro.2006.07.017

15 Mazzocca AD, Bollier MJ, Ciminiello AM, et al. Biomechanical evaluation of arthroscopic rotator cuff repairs over time. Arthroscopy 2010;26(05):592-599. Doi: 10.1016/j. arthro.2010.02.009 Erratum in: Arthroscopy. 2010; 26(6): 867

16 Higgins JPT, Green S. 2011 Cochrane handbook for systematic reviews of interventions. Version 5.1.9 [updated March 2011]. The Cochrane Collaboration

17 Moher D, Liberati A, Tetzlaff J, Altman DGPRISMA Group. Preferred reporting items for systematic reviews and metaanalyses: the PRISMA statement. PLoS Med 2009;6(07): e1000097. Doi: 10.1371/journal.pmed.1000097

18 Caldow J, Richardson M, Balakrishnan S, Sobol T, Lee PV, Ackland DC. A cruciate suture technique for rotator cuff repair. Knee Surg Sports Traumatol Arthrosc 2015;23(02):619-626. Doi: 10.1007| s00167-014-3474-7

19 Dyrna F, Beitzel K, Pauzenberger L, et al. A Superolaterally Placed Anchor for Subscapularis "Leading-Edge" Refixation: A Biomechanical Study. Arthroscopy 2019;35(05):1306-1313.e1. Doi: 10.1016/j.arthro.2018.11.060

20 Huntington L, Coles-Black J, Richardson M, et al. The use of suturetape and suture-wire in arthroscopic rotator cuff repair: A comparative biomechanics study. Injury 2018;49(11): 2047-2052. Doi: 10.1016/j.injury.2018.09.004

21 Kim SJ, Kim SH, Moon HS, Chun YM. Footprint Contact Area and Interface Pressure Comparison Between the Knotless and KnotTying Transosseous-Equivalent Technique for Rotator Cuff Repair. Arthroscopy 2016;32(01):7-12. Doi: 10.1016/j. arthro.2015.07.004

22 Liu RW, Lam PH, Shepherd HM, Murrell GAC. Tape Versus Suture in Arthroscopic Rotator Cuff Repair: Biomechanical Analysis and Assessment of Failure Rates at 6 Months. Orthop J Sports Med 2017;5(04):2325967117701212. $10.1177 / 2325967117701212$

23 Liu VK, Bouwmeester TM, Smith GCS, Lam PH. Biomechanical comparison of knotless wide suture double-row SutureBridge rotator cuff repair to double-row standard suture repair. J Shoulder Elbow Surg 2020;29(08):1621-1626

$24 \mathrm{Ng}$ SHA, Tan CHJ. Double-row repair of rotator cuff tears: Comparing tendon contact area between techniques. World J Orthop 2020;11(01):10-17. Doi: 10.5312/wjo.v11.i1.10

25 Park JS, McGarry MH, Campbell ST, et al. The optimum tension for bridging sutures in transosseous-equivalent rotator cuff repair: a cadaveric biomechanical study. Am J Sports Med 2015;43(09): 2118-2125. Doi: 10.1177/0363546515590596

26 Park MC, Peterson AB, McGarry MH, Park CJ, Lee TQ. Knotless Transosseous-Equivalent Rotator Cuff Repair Improves Biomechanical Self-reinforcement Without Diminishing Footprint Contact Compared With Medial Knotted Repair.
Arthroscopy 2017;33(08):1473-1481. Doi: 10.1016/j. arthro.2017.03.021

27 Pauzenberger L, Heuberer PR, Dyrna F, et al. Double-Layer Rotator Cuff Repair: Anatomic Reconstruction of the Superior Capsule and Rotator Cuff Improves Biomechanical Properties in Repairs of Delaminated Rotator Cuff Tears. Am J Sports Med 2018;46(13): 3165-3173. Doi: $10.1177 / 0363546518796818$

28 Simmer Filho J, Voss A, Pauzenberger L, et al. Footprint coverage comparison between knotted and knotless techniques in a singlerow rotator cuff repair: biomechanical analysis. BMC Musculoskelet Disord 2019;20(01):123. Doi: 10.1186/s12891019-2479-2

29 Smith GCS, Lam PH. Shoulder abduction diminishes selfreinforcement in transosseous-equivalent rotator cuff repair in both knotted and knotless techniques. Knee Surg Sports Traumatol Arthrosc 2018;26(12):3818-3825. Doi: 10.1007/ s00167-018-4999-y

30 Smith GCS, Bouwmeester TM, Lam PH. Knotless double-row SutureBridge rotator cuff repairs have improved selfreinforcement compared with double-row SutureBridge repairs with tied medial knots: a biomechanical study using an ovine model. J Shoulder Elbow Surg 2017;26(12):2206-2212. Doi: $10.1016 /$ j.jse.2017.06.045

31 Stone AV, Luo TD, Sharma A, Danelson KA, De Gregorio M, Freehill MT. Optimizing the Double-Row Construct: An Untied Medial Row Demonstrates Equivalent Mean Contact Pressures in a Rotator Cuff Model. Orthop J Sports Med 2020;8(04): 2325967120914932. Doi: 10.1177/2325967120914932

32 Urch E, Lin CC, Itami Y, et al. Improved Rotator Cuff Footprint Contact Characteristics With an Augmented Repair Construct Using Lateral Edge Fixation. Am J Sports Med 2020;48(02): 444-449. Doi: 10.1177/0363546519888182

33 Rhee YG, Cho NS, Parke CS. Arthroscopic rotator cuff repair using modified Mason-Allen medial row stitch: knotless versus knottying suture bridge technique. Am J Sports Med 2012;40(11): 2440-2447. Doi: 10.1177/0363546512459170

34 Mall NA, Lee AS, Chahal J, et al. Transosseous-equivalent rotator cuff repair: a systematic review on the biomechanical importance of tying the medial row. Arthroscopy 2013;29(02):377-386. Doi: 10.1016/j.arthro.2012.11.008

35 Burkhart SS, Adams CR, Burkhart SS, Schoolfield JD. A biomechanical comparison of 2 techniques of footprint reconstruction for rotator cuff repair: the SwiveLock-FiberChain construct versus standard double-row repair. Arthroscopy 2009; 25(03):274-281. Doi: 10.1016/j.arthro.2008.09.024

36 Christoforetti JJ, Krupp RJ, Singleton SB, Kissenberth MJ, Cook C, Hawkins RJ. Arthroscopic suture bridge transosseus equivalent fixation of rotator cuff tendon preserves intratendinous blood flow at the time of initial fixation. J Shoulder Elbow Surg 2012;21 (04):523-530. Doi: 10.1016/j.jse.2011.02.012

37 Liem D, Dedy NJ, Hauschild G, et al. In vivo blood flow after rotator cuff reconstruction in a sheep model: comparison of single versus double row. Knee Surg Sports Traumatol Arthrosc 2015;23(02): 470-477. Doi: 10.1007/s00167-013-2429-8

38 Bisson LJ, Manohar LM. A biomechanical comparison of the pullout strength of No. 2 FiberWire suture and 2-mm FiberWire tape in bovine rotator cuff tendons. Arthroscopy 2010;26(11):1463-1468. Doi: 10.1016/j.arthro.2010.04.075

39 De Carli A, Lanzetti RM, Monaco E, Labianca L, Mossa L, Ferretti A. The failure mode of two reabsorbable fixation systems: Swivelock with Fibertape versus Bio-Corkscrew with Fiberwire in bovine rotator cuff. J Orthop Sci 2012;17(06):789-795. Doi: 10.1007/ s00776-012-0275-z Erratum in: J Orthop Sci. 2012; 17(6): 830

40 Frank JB, ElAttrache NS, Dines JS, Blackburn A, Crues J, Tibone JE. Repair site integrity after arthroscopic transosseous-equivalent suture-bridge rotator cuff repair. Am J Sports Med 2008;36(08): 1496-1503. Doi: 10.1177/0363546507313574 
41 Lafosse L, Jost B, Reiland Y, Audebert S, Toussaint B, Gobezie R. Structural integrity and clinical outcomes after arthroscopic repair of isolated subscapularis tears. J Bone Joint Surg Am 2007;89(06):1184-1193. Doi: 10.2106/JBJS.F.00007

42 Yoo JC, Rhee YG, Shin SJ, et al. Subscapularis tendon tear classification based on 3-dimensional anatomic footprint: a cadaveric and prospective clinical observational study. Arthroscopy 2015;31(01):19-28. Doi: 10.1016/j. arthro.2014.08.015

43 Sonnabend DH, Yu Y, Howlett CR, Harper GD, Walsh WR. Laminated tears of the human rotator cuff: a histologic and immunochemical study. J Shoulder Elbow Surg 2001;10(02): 109-115. Doi: 10.1067/mse.2001.112882

44 Nimura A, Kato A, Yamaguchi K, et al. The superior capsule of the shoulder joint complements the insertion of the rotator cuff. J Shoulder Elbow Surg 2012;21(07):867-872. Doi: 10.1016/j. jse.2011.04.034

45 Boileau P, Brassart N, Watkinson DJ, Carles M, Hatzidakis AM, Krishnan SG. Arthroscopic repair of full-thickness tears of the supraspinatus: does the tendon really heal? J Bone Joint Surg Am 2005;87(06):1229-1240. Doi: 10.2106/JBJS.D.02035
46 Baleani M, Ohman C, Guandalini L, et al. Comparative study of different tendon grasping techniques for arthroscopic repair of the rotator cuff. Clin Biomech (Bristol, Avon) 2006;21(08): 799-803. Doi: 10.1016/j.clinbiomech.2006.04.011

47 Ma CB, Comerford L, Wilson J, Puttlitz CM. Biomechanical evaluation of arthroscopic rotator cuff repairs: double-row compared with single-row fixation. J Bone Joint Surg Am 2006; 88(02):403-410. Doi: 10.2106/JBJS.D.02887

48 Park MC, McGarry MH, Gunzenhauser RC, Benefiel MK, Park CJ, Lee TQ. Does transosseous-equivalent rotator cuff repair biomechanically provide a "self-reinforcement" effect compared with single-row repair? J Shoulder Elbow Surg 2014;23(12): 1813-1821. Doi: 10.1016/j.jse.2014.03.008

49 Mook WR, Greenspoon JA, Millett PJ. Arthroscopic Double-Row Transosseous Equivalent Rotator Cuff Repair with a Knotless SelfReinforcing Technique. Open Orthop J 2016;10:286-295. Doi: $10.2174 / 1874325001610010286$

$50 \mathrm{Kim}$ SH, Kim J, Choi YE, Lee HR. Healing disturbance with suture bridge configuration repair in rabbit rotator cuff tear. J Shoulder Elbow Surg 2016;25(03):478-486. Doi: 10.1016/j. jse.2015.08.035 